\title{
Hybrid Geodesics as Optimal Solutions to the Collision-Free Motion Planning Problem ${ }^{\star}$
}

\author{
Jianghai $\mathrm{Hu}^{1}$, Maria Prandini ${ }^{2}$, Karl Henrik Johansson ${ }^{3}$, and Shankar Sastry ${ }^{1}$ \\ 1 Dept. of Electrical Engineering and Computer Sciences \\ University of California at Berkeley - Berkeley CA 94720, USA \\ \{jianghai, sastry\}@eecs. berkeley.edu \\ 2 Dept. of Electrical Engineering for Automation \\ University of Brescia - Brescia, Italy \\ prandini@ing.unibs.it \\ 3 Dept. of Signals, Systems and Sensors \\ Royal Institute of Technology - Stockholm, Sweden \\ kallej@s3.kth.se
}

\begin{abstract}
In this paper we address the problem of designing energy minimizing collision-free maneuvers for multiple agents moving on a plane. We show that the problem is equivalent to that of finding the shortest geodesic in a certain manifold with nonsmooth boundary. This allows us to prove that the optimal maneuvers are $C^{1}$ by introducing the concept of $u$-convex manifolds. Moreover, due to the nature of the optimal maneuvers, the problem can be formulated as an optimal control problem for a certain hybrid system whose discrete states consist of different "contact graphs". We determine the analytic expression for the optimal maneuvers in the two agents case. For the three agents case, we derive the dynamics of the optimal maneuvers within each discrete state. This together with the fact that an optimal maneuver is a $C^{1}$ concatenation of segments associated with different discrete states gives a characterization of the optimal solutions in the three agents case.
\end{abstract}

\section{Introduction and background}

Many problems arising in practical situations have boundary constraints and can be described in the setting of manifolds with boundary. Here we are interested in certain geometric aspects of such manifolds, specifically those concerning the properties of geodesics, i.e., locally distance minimizing curves. It is intuitively clear that when the boundary consists of cells of various dimensions pieced together, a geodesic is in general "hybrid" in the sense that it is a concatenation of different segments, each one of which being a geodesic of a particular cell (in its own geometry). Thus in the hybrid systems terminology ([16]), the geodesics can be naturally described as the executions of an underlying hybrid system.

\footnotetext{
* Research supported by NSF and DARPA. The authors would like to thank Ekaterina Lemch for the helpful discussions.
} 
Note that our interpretation of a manifold with boundary as the domain for the continuous state of a hybrid system is the inverse of the procedure adopted in [21], where the concept of hybrifold is introduced by piecing together the domains corresponding to all the discrete modes of a hybrid system to form a single topological manifold. Another difference is that, in addition to the topological properties of the hybrid systems such as stability, zenoness, ergodicity, etc., we are also interested in their metric properties such as distance, curve length, angle, etc. Therefore when piecing domains together, isometries instead of merely diffeomorphisms are required as the identifying maps of the boundaries.

To be precise, let $M$ be a connected $m$-dimensional $C^{\infty}$ Riemannian manifold with boundary. The boundary of $M$ can be either smooth or nonsmooth. Consider only those curves in $M$ which are piecewise $C^{1}$, i.e., curves which can be partitioned into a countable number of $C^{1}$ segments. For such curves the arc length is well defined. The distance between two points in $M$ is then defined as the infimum of the arc length of all the piecewise $C^{1}$ curves connecting them. A geodesic in $M$ is a locally distance minimizing curve. More precisely, the curve $\gamma:\left(t_{0}, t_{f}\right) \rightarrow M$ is a geodesic if and only if for each $t \in\left(t_{0}, t_{f}\right), \gamma$ is the shortest curve between $\gamma\left(t_{1}\right)$ and $\gamma\left(t_{2}\right)$ for every $t_{1}, t_{2}$ belonging to a neighborhood of $t$ with $t_{1}<t<t_{2}$. Given two arbitrary points in $M$, the (globally) shortest curve connecting them is automatically a geodesic. However, it is well known that the converse is not true: a geodesic is not necessarily distance minimizing between its end points. In fact, even for manifolds without boundary, a geodesic is no longer distance minimizing after its first conjugate point ([5]).

Due to the presence of the boundary, regularity of geodesics in $M$ is an issue. The special case of geodesics in manifolds with smooth boundary is dealt with in $[2,3]$, to name a few. We now review briefly some of the results in these papers relevant to our study in the nonsmooth boundary case. For manifolds with smooth boundary, it is shown in [3] that geodesics are in general $C^{1}$ but not $C^{2}$. The simplest example is $\mathbb{R}^{2}$ with a unit disk removed. Two points across the disk and "invisible" to each other are connected by at most two shortest geodesics, which are $C^{1}$ everywhere but fail to be $C^{2}$ at exactly the points where geodesics switch from a line segment to a boundary arcs or vice versa. In [3] it is further suggested that a geodesic in a manifold $M$ with smooth boundary can be decomposed into: (1) interior segments, which are geodesic segments belonging to the interior of $M ;(2)$ boundary segments, which are geodesic segments belonging to the boundary $\partial M$ of $M ;(3)$ switch points, which are points where the geodesic switches from a boundary segment to an interior segment or vice versa; (4) intermittent points, which are accumulation points of the set of switch points. It is proved in [1] that when the boundary $\partial M$ is locally analytic, a geodesic can have only a finite number of switch points in any segment of finite arc length, hence no intermittent points at all. In our interpretation of geodesics as the executions of an underlying hybrid system, switch points correspond to transitions between discrete states, and the existence of intermittent points in a geodesic implies that the corresponding execution, hence the hybrid system, is Zeno ([16]). Therefore 
the result in [1] can be rephrased by saying that a hybrid system whose executions correspond to geodesics in a manifold with locally analytic boundary is non-Zeno.

In this paper we study the problem of optimal collision-free motion planning for multiple agents moving on a plane, where a collision is the event that any two agents get closer than a minimum allowed distance. We show that each collisionfree joint maneuver has a natural representation as a curve in a certain manifold with boundary, and among all such joint maneuvers the one with the least energy corresponds to a geodesic parameterized proportionally to arc length. Geodesics satisfying this property are called normalized. Unless otherwise stated, we assume throughout the paper that all geodesics are normalized.

The problem which inspired this work originally is the development of algorithms for aircraft conflict resolution. Aircraft flying at the same altitude must maintain a horizontal separation of at least 3 nautical miles (nmi) inside the terminal radar approach control facilities and $5 \mathrm{nmi}$ in the en-route airspace ([20]). Moreover, the energy of an aircraft maneuver is closely related to practical aspects such as travel distance, fuel consumption, passenger comfort, etc. Numerous approaches have been suggested in the literature to deal with aircraft conflict resolution (see the survey paper [13]). Some of them $([6,8,11,17])$ actually pose the problem as a constrained optimization problem. In particular, in [11] the geometric interpretation of aircraft motions as a braid is used in performing the optimality analysis. Optimal multi-agent coordination also finds applications in other transportation systems, for example [18]. Another related field is the motion planning for mobile robots. Most of the papers in this field focus on the feasibility and the algorithmic complexity aspect of the problem $([7,9,14,22])$. Among those dealing with optimal coordination, [15] considers the case when each robot minimizes its own independent goal by using techniques from multiobjective optimization and game theory. [4] studies the problem of time-optimal control of multiple vehicles moving on a plane with constant speed and bounded curvature.

The rest of the paper is organized as follows. In Sect. 2, we describe the optimal collision-free motion planning problem and show how it can be reformulated as the problem of finding the shortest geodesic in a manifold $M$ with nonsmooth boundary. Using the fact that $M$ is a u-convex manifold, we are able to prove in Sect. 2.2 that the optimal motions for the agents are $C^{1}$. We then introduce in Sect. 3 the notion of "contact graph", which leads to a natural interpretation of the problem in the framework of optimal control for a certain hybrid system. The $C^{1}$ property implies that the reset maps of the hybrid system are all identity maps. The shortest geodesic can be obtained by appropriately piecing together geodesic segments in different discrete modes, and is the optimal execution for the hybrid system. In Sect. 3.1 necessary conditions are introduced to simplify the determination of such geodesics, which are then used in Sect. 3.2 and 3.3 to characterize the optimal collision-free motions for the two agents and three agents case respectively. Finally some concluding remarks are given in Sect. 4. 


\section{Problem formulation}

Consider the situation when $n$ agents, numbered from 1 to $n$, are moving on a common plane $\mathbb{R}^{2}$. The $n$ agents are required to start from positions $a_{1}, \ldots, a_{n} \in$ $\mathbb{R}^{2}$ at time $t_{0}$ and reach positions $b_{1}, \ldots, b_{n} \in \mathbb{R}^{2}$ at a fixed time $t_{f}$. We assume that each one of the two sets $\left\{a_{i}\right\}_{i=1}^{n}$ and $\left\{b_{i}\right\}_{i=1}^{n}$ satisfies the $r$-separation condition for some positive $r$, in the sense that the minimum pairwise Euclidean distance in each set is at least $r$.

A maneuver for agent $i, 1 \leq i \leq n$, is defined to be a piecewise $C^{1}$ map $\alpha_{i}:\left[t_{0}, t_{f}\right] \rightarrow \mathbb{R}^{2}$ satisfying $\alpha_{i}\left(t_{0}\right)=a_{i}$ and $\alpha_{i}\left(t_{f}\right)=b_{i}$. The set of all maneuvers for agent $i$ is denoted as $\mathcal{P}_{i}$. Then $\mathcal{P}=\prod_{i=1}^{n} \mathcal{P}_{i}$ is the set of joint maneuvers for the $n$-agent system. Here we are interested in the subset $\mathcal{P}(r)$ of $\mathcal{P}$ consisting of all the collision-free maneuvers, i.e., those joint maneuvers $\alpha=\left(\alpha_{1}, \ldots, \alpha_{n}\right) \in \mathcal{P}$ such that $\left\{\alpha_{i}(t)\right\}_{i=1}^{n}$ satisfies the $r$-separation condition at each time $t, t \in\left[t_{0}, t_{f}\right]$.

The energy of a joint maneuver $\alpha=\left(\alpha_{1}, \ldots, \alpha_{n}\right) \in \mathcal{P}$ is defined as

$$
J(\alpha) \triangleq \frac{1}{2} \sum_{i=1}^{n} \int_{t_{0}}^{t_{f}}\left\|\dot{\alpha}_{i}(t)\right\|^{2} d t
$$

The goal is to find the collision-free maneuver $\alpha \in \mathcal{P}(r)$ with minimal energy. This leads to the following formulation of the problem:

$$
\text { Minimize } J(\alpha) \text { subject to } \alpha \in \mathcal{P}(r) \text {. }
$$

Notice that in formulating problem (1), we make the restrictive assumption that all the agents involved in the encounter reach their destinations at the same known time instant $t_{f}$. This is important in time-critical applications such as air traffic management. The issue of choosing $t_{f}$ is not dealt with in this paper.

Remark 1. Problem (1) can be alternatively formulated as an optimal control problem with state constraints, and approached by using the corresponding techniques from optimal control theory. In this paper, however, we adopt a geometric point of view. The geometric method not only yields elegant results and proofs, but more importantly, by using information on the curvature of the domains, it also allows us to obtain deeper results concerning the global uniqueness of the optimal solutions under certain conditions (see [12]).

\subsection{A geometric interpretation}

Each joint maneuver $\alpha=\left(\alpha_{1}, \ldots, \alpha_{n}\right)$ in $\mathcal{P}$ can be re-interpreted as a curve in $\mathbb{R}^{2 n}$ defined by $\alpha(t)=\left(\alpha_{1}(t), \ldots, \alpha_{n}(t)\right), t \in\left[t_{0}, t_{f}\right]$, which starts from $a=$ $\left(a_{1}, \ldots, a_{n}\right)$ and ends at $b=\left(b_{1}, \ldots, b_{n}\right)$. If we use $\left(x_{1}, y_{1}, \ldots, x_{n}, y_{n}\right)$ to denote the coordinates of a generic point in $\mathbb{R}^{2 n}$, then the collision-free constraint on the joint maneuver $\alpha$ translates into the condition that $\alpha$ viewed as a curve in $\mathbb{R}^{2 n}$ is strictly contained in $M$, a manifold with boundary obtained by removing from $\mathbb{R}^{2 n}$ the "static obstacle" $W$ given by

$$
W=\left\{\mathrm{P} \in \mathbb{R}^{2 n}: \sqrt{\left(x_{i}-x_{j}\right)^{2}+\left(y_{i}-y_{j}\right)^{2}}<r \text { for some } 1 \leq i<j \leq n\right\} .
$$


In other words, $M=\mathbb{R}^{2 n} \backslash W$. Thus there is a one-to-one correspondence between maneuvers in $\mathcal{P}(r)$ and piecewise $C^{1}$ curves in $M$ connecting $a$ and $b$. Moreover, the energy of a joint maneuver $\alpha=\left(\alpha_{1}, \ldots, \alpha_{n}\right) \in \mathcal{P}$ can be expressed as $J(\alpha)=\frac{1}{2} \sum_{i=1}^{n} \int_{t_{0}}^{t_{f}}\left\|\dot{\alpha}_{i}(t)\right\|^{2} d t=\frac{1}{2} \int_{t_{0}}^{t_{f}}\|\dot{\alpha}(t)\|^{2} d t$, which coincides with the usual definition of the energy of $\alpha$ viewed as a curve in $\mathbb{R}^{2 n}$. Hence (1) is equivalent to the following geometric problem:

Find the energy minimizing curve $\alpha$ in $M$ joining point $a$ to point $b$.

It is a standard result (see, e.g., [19]) that solutions to (3) are shortest curves in $M$ from $a$ to $b$ parameterized proportionally to arc length, i.e., minimizing geodesics in $M$ connecting $a$ to $b$. We shall henceforth study problem (3) instead of (1) with the understanding that all the curves connecting $a$ to $b$ in $M$ are parameterized so that they start from $a$ at $t_{0}$ and end at $b$ at $t_{f}$.

Notice that $W$ defined in (2) is the union of $n(n-1) / 2$ convex open cylinders, each one of the form $\left\{\left(x_{1}, y_{1}, \ldots, x_{n}, y_{n}\right): \sqrt{\left(x_{i}-x_{j}\right)^{2}+\left(y_{i}-y_{j}\right)^{2}}<r\right\}$ for some $(i, j)$, with $i \neq j$. Therefore $M$ obtained by removing $W$ from $R^{2 n}$ is an instance of the following class of manifolds with boundary:

Definition 1 (u-convex manifolds). A manifold with boundary is called uconvex if it is obtained by removing from some Euclidean space $\mathbb{R}^{m}$ a finite union of open convex subsets, each one of which has a smooth boundary.

We will show in the next section that geodesics in u-convex manifolds are $C^{1}$, which implies that solutions to problem (3), hence (1), are $C^{1}$.

\subsection{Geodesics in u-convex manifolds}

In this section we study the properties of geodesics in u-convex manifolds. Many technicalities encountered in the general case can be avoided when analyzing this special case. For example, when the boundary of $M$ is nonsmooth, geodesics in $M$ are in general not $C^{1}$ since they can bend into sharp corners of the boundary. However, we next show that this is not the case for u-convex manifolds.

Suppose $M$ is u-convex, i.e., $M=\mathbb{R}^{m} \backslash \cup_{i=1}^{k} D_{i}$ is the complement in $\mathbb{R}^{m}$ of the union of open convex bodies $D_{1}, \ldots, D_{k} \subset \mathbb{R}^{m}$, whose boundary $\partial D_{i}$ is smooth for each $i=1, \ldots, k$. Then at each point $x \in M$, we can define the visible cone of $x$ to be the cone $V(x)$ with vertex $x$ and consisting of all the rays which start from $x$ and lie inside $M$ within a sufficiently small distance. In other words, $V(x)$ is the region a viewer sitting at $x$ can see if only local obstacles around $x$ are considered. $V(x)$ can be obtained in the following way. If $x \in M$ lies on the boundary of $D_{i}$ for exactly those $i$ belonging to a subset $\mathcal{I}$ of $\{1, \ldots, k\}$, then the obstacles $D_{i}, i \in \mathcal{I}$, are called the active obstacles at $x$. For each active obstacle $D_{i}$, let $T_{x}\left(\partial D_{i}\right)$ be the plane tangent to $\partial D_{i}$ at $x$ and $n_{i}$ be the unit normal vector of $\partial D_{i}$ at $x$ pointing outside of $D_{i} . T_{x}\left(\partial D_{i}\right)$ separates $\mathbb{R}^{m}$ into two open

half spaces. We denote the one containing $n_{i}$ as $P_{i, x}^{+}$and its closure as $\overline{P_{i, x}^{+}}$. The convexity of $D_{i}$ implies that $P_{i, x}^{+}$and $D_{i}$ are disjoint sets. Then $V(x)$ is given by 

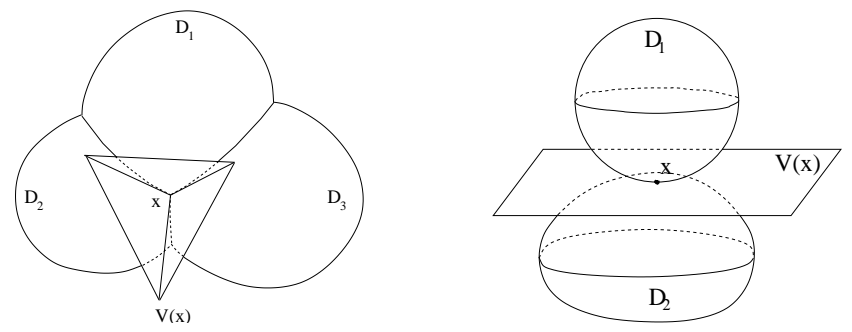

Fig. 1. Examples of visible cones. On the right a degenerate case.

$V(x)=\bigcap_{i \in \mathcal{I}} \overline{P_{i, x}^{+}} . V(x)$ is a closed convex cone since it is the finite intersection of closed convex sets (half spaces), and it can have an arbitrary dimension lower than $m$. Figure 1 shows examples of visible cones in $\mathbb{R}^{3}$. In the case when $x$ is in the interior of $M, V(x)=\mathbb{R}^{m}$ since there are no active obstacles at $x$.

By using the notion of visible cone, one can prove the following result.

Theorem 1. Suppose that $M$ is u-convex. Then any geodesic in $M$ is $C^{1}$.

Proof. Let $\gamma: I \rightarrow M$ be a geodesic of $M$, where $I$ is an open interval in $\mathbb{R}$. For each $s \in I$, the one-sided derivatives $\gamma^{\prime}\left(s^{-}\right)$and $\gamma^{\prime}\left(s^{+}\right)$of $\gamma$ at $s$ exist since $\gamma$ is piecewise $C^{1}$. By using a reparameterization if necessary, we can assume that both of them are unit vectors. Construct the visible cone $V(x)$ of $x=\gamma(s)$. By definition, both $\gamma^{\prime}\left(s^{+}\right)$and $-\gamma^{\prime}\left(s^{-}\right)$based at $x$ lie inside $V(x)$ and they span an angle $\theta \in[0, \pi]$. Suppose by contradiction that $\gamma^{\prime}\left(s^{-}\right) \neq \gamma^{\prime}\left(s^{+}\right)$, then $\theta<\pi$.

Fix a neighborhood $U$ of $x$ small enough so that only the active obstacles at $x$ intersect $U$. Choose $\epsilon$ such that $\left.\gamma\right|_{[s-\epsilon, s+\epsilon]} \subset U$. For each $t \in[s-\epsilon, s]$, let $\hat{\gamma}(t)$ be the projection of $\gamma(t)$ onto the line through $x$ and along the direction $-\gamma^{\prime}\left(s^{-}\right)$; for each $t \in[s, s+\epsilon]$, let $\hat{\gamma}(t)$ be the projection of $\gamma(t)$ onto the line through $x$ and along the direction $\gamma^{\prime}\left(s^{+}\right)$. Notice that $\left.\hat{\gamma}\right|_{[s-\epsilon, s+\epsilon]}$ is a curve through $x$ contained completely within $M$. By choosing $\epsilon$ small enough, one can ensure that the line segments $\overline{\gamma(s-\epsilon) \hat{\gamma}(s-\epsilon)}$ and $\overline{\gamma(s+\epsilon) \hat{\gamma}(s+\epsilon)}$ both lie completely inside $M$. Therefore by replacing the arc $\left.\gamma\right|_{[s-\epsilon, s+\epsilon]}$ with the concatenation of $\overline{\gamma(s-\epsilon) \hat{\gamma}(s-\epsilon)}$, the arc $\left.\hat{\gamma}\right|_{[s-\epsilon, s+\epsilon]}$, and $\overline{\hat{\gamma}(s+\epsilon) \gamma(s+\epsilon)}$, the total arc length is increased by at most $o(\epsilon)$. Notice further that we can shortcut $\left.\hat{\gamma}\right|_{[s-\epsilon, s+\epsilon]}$ by the line segment $\bar{\gamma}(s-\epsilon) \hat{\gamma}(s+\epsilon)$, which lies completely inside $V(x)$ (hence $M$ ) by the convexity of $V(x)$. Doing so can reduce the arc length of $\left.\hat{\gamma}\right|_{[s-\epsilon, s+\epsilon]}$ by at least $2 \epsilon(1-\sin (\theta / 2))+o(\epsilon)$, where we use the fact that $\gamma^{\prime}\left(s^{-}\right)$ and $\gamma^{\prime}\left(s^{+}\right)$are unit vectors. Therefore the concatenation of the line segments $\overline{\gamma(s-\epsilon) \hat{\gamma}(s-\epsilon)}, \overline{\hat{\gamma}(s-\epsilon) \hat{\gamma}(s+\epsilon)}$, and $\overline{\hat{\gamma}(s+\epsilon) \gamma(s+\epsilon)}$ is a curve in $M$ shorter than the arc $\left.\gamma\right|_{[s-\epsilon, s+\epsilon]}$ for $\epsilon$ small enough. This contradicts the fact that $\gamma$ is locally distance minimizing. Thus $\theta=\pi$ and $\gamma$ is $C^{1}$ everywhere.

To show the necessity of u-convexity in proving Theorem 1, we plot in Fig. 2 an example in which $M$ is obtained by removing from $\mathbb{R}^{3}$ a nonconvex obstacle given by the exterior $D_{1}$ of a cylinder with axis $l_{1}$ and a convex obstacle given by 


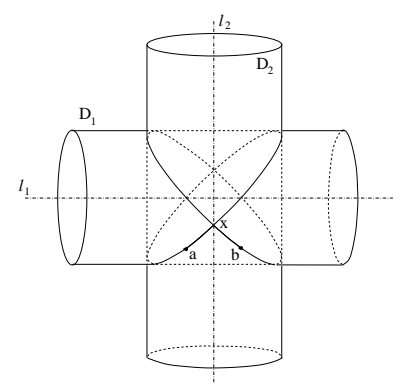

Fig. 2. Geodesic in a manifold with boundary that is not u-convex.

the interior $D_{2}$ of a cylinder with the same radius and with axis $l_{2}$ intersecting $l_{1}$ at a right angle. Hence $M$ consists of all those points in $\mathbb{R}^{3}$ which lie inside the cylinder with axis $l_{1}$ but outside the cylinder with axis $l_{2}$, with the points on their boundaries included. The heavy-weighted curve in Fig. 2 is a geodesic in $M$ with end points $a$ and $b$, which is clearly not $C^{1}$ at $x$.

\section{Hybrid system solution}

Now we go back to the discussion of the optimization problem (3) proposed in Sect. 2, where $M=\mathbb{R}^{2 n} \backslash W$ with $W$ defined in (2).

Consider a curve $\alpha=\left(\alpha_{1}, \ldots, \alpha_{n}\right)$ from $a$ to $b$ in $M$ corresponding a collisionfree maneuver in $\mathcal{P}(r)$. Fix a time instant $t \in\left[t_{0}, t_{f}\right]$. We say that agent $i$ and agent $j$ contact at time $t$ if and only if $\left\|\alpha_{i}(t)-\alpha_{j}(t)\right\|=r$. A graph can be associated to $\alpha$ at time $t$ in the following way. The graph has $n$ vertices, numbered from 1 to $n$, each one corresponding to an agent, and an edge exists between vertex $i$ and vertex $j$ if and only if agent $i$ and agent $j$ contact at time $t$. We call this graph the contact graph of $\alpha$ at time $t$.

Let $\alpha^{*}$ be a curve from $a$ to $b$ in $M$ that is a solution to problem (3). Suppose that there is a finite subdivision of $\left[t_{0}, t_{f}\right]: t_{0} \leq t_{1} \leq \ldots \leq t_{k-1} \leq t_{k}=t_{f}$, such that the contact graph of $\alpha^{*}$ over the subinterval $\left(t_{h-1}, t_{h}\right)$ (which we denote as $G_{h}$ ) remains constant for all $h=1, \ldots, k$, while contiguous subintervals have distinct contact graphs. In each subinterval, say $\left(t_{h-1}, t_{h}\right), \alpha^{*}$ moves on a certain part of $M$ determined by $G_{h}$. If $G_{h}$ has no edges, then $\alpha^{*}$ restricted to $\left(t_{h-1}, t_{h}\right)$ is a straight line segment in the interior of $M$. If $G_{h}$ has at least one edge, then $\alpha^{*}$ restricted to $\left(t_{h-1}, t_{h}\right)$ moves on a portion of the boundary of $M$, which is a lower dimensional smooth submanifold of $\mathbb{R}^{2 n}$ consisting of all the points $\left(x_{1}, y_{1}, \ldots, x_{n}, y_{n}\right)$ in $\mathbb{R}^{2 n}$ such that $\sqrt{\left(x_{i}-x_{j}\right)^{2}+\left(y_{i}-y_{j}\right)^{2}}$ is equal to $r$ for $(i, j)$ such that there is an edge between vertices $i$ and $j$ in $G_{h}$, and greater than $r$ for all others $(i, j), i \neq j$. Moreover, $\alpha^{*}$ restricted to $\left(t_{h-1}, t_{h}\right)$ is a minimizing geodesic in this submanifold. In this way we can associate to each type of contact graph a domain, i.e., the submanifold of $M$ to which $\alpha^{*}$ belongs when its contact graph is of that type. 
Based on the above analysis, $\alpha^{*}$ can be viewed as an execution of a certain hybrid system, whose continuous variable takes values in $M$, and whose discrete modes have a one-to-one correspondence with the different contact graphs for the $n$-agent system. For each discrete mode, the invariant set is the domain of the corresponding contact graph, and the dynamics is governed by the geodesic equation on that domain, which is a second-order ordinary differential equation. By Theorem 1, when a transition occurs between discrete modes, the position $\alpha$ and the velocity $\dot{\alpha}$ are reset by identity maps. $\alpha$ is an optimal solution to this hybrid system if it satisfies $\alpha\left(t_{0}\right)=a$ and $\alpha\left(t_{f}\right)=b$, and has minimal energy. The problem is to determine the initial velocity $\dot{\alpha}\left(t_{0}\right)$ and the time and sequence of the discrete switchings so that the corresponding execution of this hybrid system will generate the optimal solution.

\subsection{Necessary conditions for optimality}

We now derive some necessary conditions for $\alpha$ to be an optimal solution to problem (3), which can then be used to simplify the determination of optimal maneuvers for the two-agent and three-agent cases.

Proposition 1. Suppose that $\alpha^{*}$ is a minimizing geodesic from a to $b$ in $M$. Fix an arbitrary $w \in \mathbb{R}^{2}$. Then $\beta^{*}=\left(\beta_{1}^{*}, \ldots, \beta_{n}^{*}\right)$ defined by

$$
\beta_{i}^{*}(t)=\alpha_{i}^{*}(t)+w \frac{t-t_{0}}{t_{f}-t_{0}}, \quad t \in\left[t_{0}, t_{f}\right], \quad i=1, \ldots, n,
$$

is a minimizing geodesic from a to $b^{\prime}=\left(b_{1}+w, \ldots, b_{n}+w\right)$ in $M$.

Proof. For each curve $\beta$ from $a$ to $b^{\prime}$ in $M$, define curve $\alpha=\left(\alpha_{1}, \ldots, \alpha_{n}\right)=$ $\mathcal{T}_{-w}(\beta)$ in $\mathbb{R}^{2 n}$ as $\alpha_{i}(t)=\beta_{i}(t)-w \frac{t-t_{0}}{t_{f}-t_{0}}$, for $t \in\left[t_{0}, t_{f}\right]$ and $i=1, \ldots, n$. Then it is easily verified that $\alpha$ is a curve in $M$ from $a$ to $b$ with energy

$$
J(\alpha)=J(\beta)+\frac{w^{T}\left(\sum_{i=1}^{n}\left(a_{i}-b_{i}\right)-n w / 2\right)}{t_{f}-t_{0}} .
$$

The second term of the right hand side of (5) is a constant independent of $\beta$, which we shall denote as $C$. From (5) and the optimality of $\alpha^{*}$, we have $J(\beta)=J(\alpha)-C \geq J\left(\alpha^{*}\right)-C=J\left(\beta^{*}\right)$, where the last equality follows by noticing that $\alpha^{*}=\mathcal{T}_{-w}\left(\beta^{*}\right)$. This is true for arbitrary $\beta$, hence the conclusion.

One important implication of Proposition 1 is that it suffices to solve problem (3) only for those $a$ and $b$ that are aligned, i.e., $a$ and $b$ with the same centroid $\frac{1}{n} \sum_{i=1}^{n} a_{i}=\frac{1}{n} \sum_{i=1}^{n} b_{i} \in \mathbb{R}^{2}$. In fact for non-aligned $a$ and $b$, by choosing $w=\sum_{i=1}^{n} a_{i}-\sum_{i=1}^{n^{n}} b_{i}$, one can ensure that $a$ and $b^{\prime}=\left(b_{1}+w, \ldots, b_{n}+w\right)$ are aligned. Hence by Proposition 1, minimizing geodesics from $a$ to $b$ can be obtained from minimizing geodesics from $a$ to $b^{\prime}$ by applying the inverse of the transformation (4). 
Proposition 2. Assume that $\alpha^{*}$ is a minimizing geodesic from a to $b$ in $M$. Then

$$
\sum_{i=1}^{n} \alpha_{i}^{*}(t)=\frac{\left(t_{f}-t\right) \sum_{i=1}^{n} a_{i}+\left(t-t_{0}\right) \sum_{i=1}^{n} b_{i}}{t_{f}-t_{0}}, \quad \forall t \in\left[t_{0}, t_{f}\right]
$$

Proof. Consider first the case when $a$ and $b$ are aligned. Define a piecewise $C^{1}$ map $g:\left[t_{0}, t_{f}\right] \rightarrow \mathbb{R}^{2}$ by $g(t)=\sum_{i=1}^{n} \alpha_{i}^{*}(t)-\sum_{i=1}^{n} a_{i}, t \in\left[t_{0}, t_{f}\right]$, which satisfies $g\left(t_{0}\right)=g\left(t_{f}\right)=0$. For each $\lambda \in \mathbb{R}$, let $\beta_{\lambda}=\left(\beta_{\lambda, 1}, \ldots, \beta_{\lambda, n}\right)$ be given by $\beta_{\lambda, i}(t)=\alpha_{i}^{*}(t)+\lambda g(t), t \in\left[t_{0}, t_{f}\right], i=1, \cdots, n$. Note that $\beta_{\lambda}$ is a piecewise $C^{1}$ curve from $a$ to $b$ in $M$ with energy

$$
J\left(\beta_{\lambda}\right)=J\left(\alpha^{*}\right)+\frac{n \lambda^{2}}{2} \int_{t_{0}}^{t_{f}}\left\|\sum_{i=1}^{n} \dot{\alpha}_{i}^{*}(t)\right\|^{2} d t+\lambda \int_{t_{0}}^{t_{f}}\left\|\sum_{i=1}^{n} \dot{\alpha}_{i}^{*}(t)\right\|^{2} d t .
$$

The difference $J\left(\beta_{\lambda}\right)-J\left(\alpha^{*}\right)$ is a quadratic function of $\lambda$ which, by the optimality of $\alpha^{*}$, must be nonnegative for all $\lambda$. Hence we have $\int_{t_{0}}^{t_{f}}\left\|\sum_{i=1}^{n} \dot{\alpha}_{i}^{*}(t)\right\|^{2} d t=0$, implying that $\sum_{i=1}^{n} \dot{\alpha}_{i}^{*}(t)=0$ for almost all $t \in\left[t_{0}, t_{f}\right]$. After integration, this leads to the desired conclusion for the aligned case. In the case when $a$ and $b$ are not aligned, the result follows from Proposition 1 by considering a minimizing geodesic in $M$ from $a$ to $b^{\prime}=\left(b_{1}+w, \ldots, b_{n}+w\right)$ with $w=\sum_{i=1}^{n} a_{i}-\sum_{i=1}^{n} b_{i}$ and noticing that $a$ and $b^{\prime}$ are aligned.

A geometric interpretation of the above results is as follows. Let $N$ be the two dimensional subspace of $\mathbb{R}^{2 n}$ spanned by vectors $(0,1, \ldots, 0,1)$ and $(1,0, \ldots, 1,0)$, and $V$ be the orthogonal complement of $N$ in $\mathbb{R}^{2 n}$ such that $\mathbb{R}^{2 n}=V \oplus N$. Then the condition that $a$ and $b$ are aligned is equivalent to the condition that $b-a$ belongs to $V$. Denote with $V_{a}$ the $(n-2)$-plane through $a$ and parallel to $V$. Then if $a$ and $b$ are aligned, they both belong to $V_{a}$, and by Proposition 2, a minimizing geodesic $\alpha^{*}$ in $M$ from $a$ to $b$ lies in $V_{a}$ completely. For non-aligned $a$ and $b$, let $b^{\prime}$ be the orthogonal projection of $b$ onto $V_{a}$. Then Proposition 1 and Proposition 2 say that a minimizing geodesic $\alpha^{*}$ between $a$ and $b$ in $M$ can be decomposed into two parts: its projection onto $V_{a}$, which is a minimizing geodesic from $a$ to $b^{\prime}$ in $V_{a} \cap M$; and its projection onto $N$, which is a straight line. These conclusions become evident under the following important observation: the obstacle $W$ defined in (2) is cylindrical in the direction of $N$, i.e., $x \in W$ if and only if $x+N \subset W$.

As a result of the above analysis, we can focus on the case when $a$ and $b$ are aligned. Without loss of generality, we assume that $a$ and $b$ both belong to $V$. Since any minimizing geodesic between such $a$ and $b$ is contained in $V$, we can effectively reduce our space of consideration from $M$ to $M_{0} \triangleq V \cap M$, which is a cross section of $M$ and two dimensions lower than $M$. This will make a difference when $n$ is relatively small.

Remark 2. Optimal maneuvers for the $\mathrm{n}$-agent system can be alternatively viewed as the outcomes of a mechanical experiment, in which $n$ particles of unit mass 


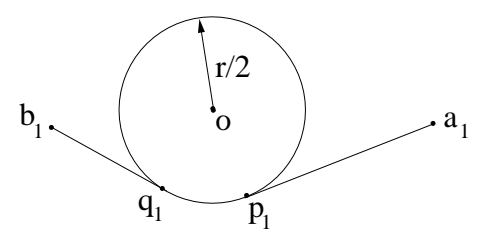

Fig. 3. Optimal $\alpha_{1}^{*}$ for two agents case.

move from positions $a_{1}, \ldots, a_{n}$ on a plane with certain initial velocities and no external force acting on them. In this interpretation, the result in Proposition 2 becomes the law of conservation of momentum. See [10] for further details.

\subsection{Two agents case}

Consider the simplest case when $n=2$ with aligned $a=\left(a_{1}, a_{2}\right)$ and $b=\left(b_{1}, b_{2}\right)$ such that $a_{1}+a_{2}=b_{1}+b_{2}=0$. If $\alpha^{*}=\left(\alpha_{1}^{*}, \alpha_{2}^{*}\right)$ is a solution to problem (3), then Proposition 2 implies that $\alpha_{1}^{*}(t)$ and $\alpha_{2}^{*}(t)$ are symmetric with respect to the origin for all $t \in\left[t_{0}, t_{f}\right]$. Hence specifying one of them, say $\alpha_{1}^{*}$, is sufficient for describing $\alpha^{*}$. Moreover, the $r$-separation constraint can be formulated as the condition that $\alpha_{1}^{*}$ can never enter the open ball $B(0, r / 2)$ of radius $r / 2$ around the origin. By noting that $\alpha_{1}^{*}$ and $\alpha_{2}^{*}$ give identical contributions to the total energy, we finally have a simplified but equivalent version of problem (3):

Find the energy minimizing curve $\alpha_{1}$ in $\mathbb{R}^{2} \backslash B(0, r / 2)$ joining $a_{1}$ to $b_{1}$.

Figure 3 shows the geometric construction of a solution $\alpha_{1}^{*}$ to problem (6), which is a geodesic of $\mathbb{R}^{2} \backslash B(0, r / 2)$ and, depending on the positions of $a_{1}$ and $b_{1}$, may contain up to three segments: first a line segment from $a_{1}$ to $p_{1}$ tangent to $\partial B(0, r / 2)$ at $p_{1}$; next from $p_{1}$ to $q_{1}$ along $\partial B(0, r / 2)$; and finally the line segment from $q_{1}$ to $b_{1}$ tangent to $\partial B(0, r / 2)$ at $q_{1}$. The case when $b_{1}$ is "visible" from $a_{1}$ is trivial.

\subsection{Three agents case}

The case $n=3$ is more complicated. Figure 4 shows all the possible contact graphs and the transitions between them, with the "ground" symbol indicating that there is a transition relation with state 1 . We now determine the geodesics in each one of the discrete states.

Geodesics in state 1. State 1 corresponds to the contact graph of three isolated vertices, hence its domain $X_{1}$ corresponds to $\operatorname{int}(M)$, the interior of $M$. By the discussion in Sect. 3.1, we only consider $X_{1}=V \cap \operatorname{int}(M)$, which has dimension 4. Geodesics in $X_{1}$ are straight lines. 


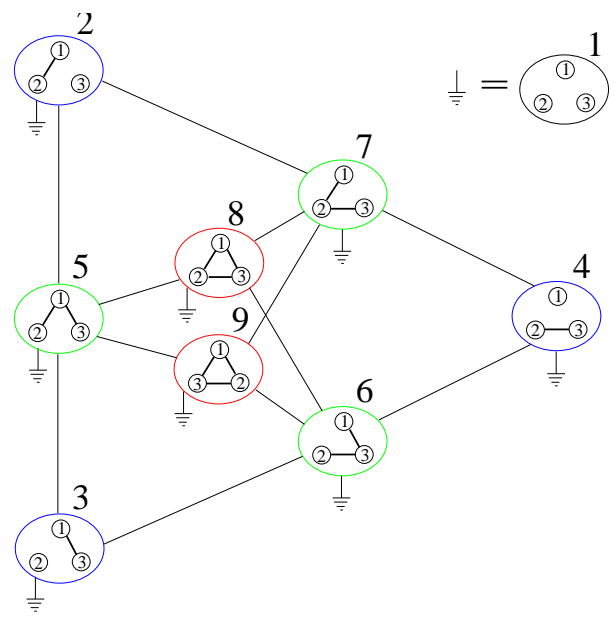

Fig. 4. State diagram.

Geodesics in state 2, 3, and 4. States 2, 3 and 4 correspond to contact graphs where two vertices are connected to each other and the third one is isolated. Let us consider state 2 . Its domain $X_{2}$ is:

$$
X_{2}=\left\{\left(x_{1}, y_{1}, x_{2}, y_{2}, x_{3}, y_{3}\right): d_{12}=r, d_{13}>r, d_{23}>r\right\} \cap V
$$

where $d_{i j} \triangleq \sqrt{\left(x_{i}-x_{j}\right)^{2}+\left(y_{i}-y_{j}\right)^{2}}$ denotes the distance between agent $i$ and agent $j . X_{2}$ has dimension 3. As long as the boundary of $X_{2}$ is not reached, a geodesic in $X_{2}$ consists of a constant velocity motion for agent 3 since it is "free", while the motions for agents 1 and 2 are determined as in Sect. 3.2 for the two agents case, followed by a possible application of Proposition 1 if their starting and destination positions are not aligned. Similarly for $X_{3}$ and $X_{4}$.

Geodesics in state 5, 6, and 7. In states 5, 6 and 7, one agent, called the pivotal agent, contacts with both the other two agents, which do not contact each other themselves. The domain for state 5 is:

$$
X_{5}=\left\{\left(x_{1}, y_{1}, x_{2}, y_{2}, x_{3}, y_{3}\right): d_{12}=r, d_{13}=r, d_{23}>r\right\} \cap V .
$$

$X_{5}$ is a 2 -dimensional submanifold with global coordinates $\left(\theta_{12}, \theta_{13}\right)$ defined by

$$
\theta_{12}=\arctan \frac{y_{2}-y_{1}}{x_{2}-x_{1}}, \quad \theta_{13}=\arctan \frac{y_{3}-y_{1}}{x_{3}-x_{1}} .
$$

$\left(\theta_{12}, \theta_{13}\right)$ takes values in $[0,2 \pi] \times[0,2 \pi]$ with opposite edges identified, i.e., the 2 -torus $T^{2}$. In order to satisfy the constraint $d_{23}>r$, the shaded region (see Fig. 5) has to be removed from $T^{2}$, resulting in a subset $\hat{X}_{5}$ homeomorphic to $S^{1} \times(0,1)$. So topologically $\hat{X}_{5}$ (hence $X_{5}$ ) is an untwisted ribbon whose boundary consists of two disjoint circles. 


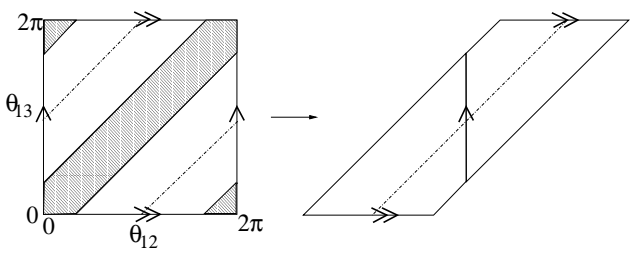

Fig. 5. The domain $X_{5}$ of discrete state 5 .

Each $\left(\theta_{12}, \theta_{13}\right) \in \hat{X}_{5}$ determines a unique point $f\left(\theta_{12}, \theta_{13}\right)$ in $X_{5}$ by

$$
\begin{aligned}
f\left(\theta_{12}, \theta_{13}\right)= & \frac{r}{3}\left(-\cos \theta_{12}-\cos \theta_{13},-\sin \theta_{12}-\sin \theta_{13}, 2 \cos \theta_{12}-\cos \theta_{13},\right. \\
& \left.2 \sin \theta_{12}-\sin \theta_{13},-\cos \theta_{12}+2 \cos \theta_{13},-\sin \theta_{12}+2 \sin \theta_{13}\right)^{T},
\end{aligned}
$$

which is an embedding of $\hat{X}_{5}$ into $\mathbb{R}^{6}$. The standard metric on $\mathbb{R}^{6}$ induces by $f$ isometrically a metric on $\hat{X}_{5}$. A curve $\left(\theta_{12}(t), \theta_{13}(t)\right)$ is a geodesic in $\hat{X}_{5}$ under the induced metric if and only if $\gamma(t)=f\left(\theta_{12}(t), \theta_{13}(t)\right)$ is a geodesic in $X_{5}$. Using the fact that $\gamma$ is a geodesic in $X_{5}$ if and only if its acceleration as a curve in $\mathbb{R}^{6}$ at each point is orthogonal to the tangent space of $X_{5}([5])$, we obtain after some calculations the geodesic equation on $\hat{X}_{5}$ as (see [11] for details)

$$
\left\{\begin{array}{l}
2 \ddot{\theta}_{12}-\cos \left(\theta_{12}-\theta_{13}\right) \ddot{\theta}_{13}=\sin \left(\theta_{12}-\theta_{13}\right)\left(\dot{\theta}_{13}\right)^{2} \\
2 \ddot{\theta}_{13}-\cos \left(\theta_{12}-\theta_{13}\right) \ddot{\theta}_{12}=-\sin \left(\theta_{12}-\theta_{13}\right)\left(\dot{\theta}_{12}\right)^{2} .
\end{array}\right.
$$

There are certain symmetries in equation (7), which become evident by writing (7) in the new coordinates $\xi=\theta_{12}+\theta_{13}$ and $\eta=\theta_{12}-\theta_{13}$, leading to:

$$
\left\{\begin{array}{l}
(2-\cos \eta) \ddot{\xi}=-\dot{\xi} \dot{\eta} \sin \eta \\
(2+\cos \eta) \ddot{\eta}=\frac{1}{2}\left((\dot{\xi})^{2}+(\dot{\eta})^{2}\right) \sin \eta
\end{array}\right.
$$

Integrating the first equation in (8), we have

$$
\dot{\xi}(2-\cos \eta)=C_{2},
$$

for some constant $C_{2}$. On the other hand, since geodesics have constant speed, there exists another constant $C_{1}$ such that ([11])

$$
(2-\cos \eta)(\dot{\xi})^{2}+(2+\cos \eta)(\dot{\eta})^{2}=4 C_{1} .
$$

Substitution of (9) into the above equation leads to

$$
(\dot{\eta})^{2}=\frac{8 C_{1}-C_{2}^{2}-4 C_{1} \cos \eta}{4-\cos ^{2} \eta}
$$

which together with (9) governs the dynamics of $\eta$ and $\xi$ respectively.

Geodesics in $X_{6}$ and $X_{7}$ can be obtained similarly. 


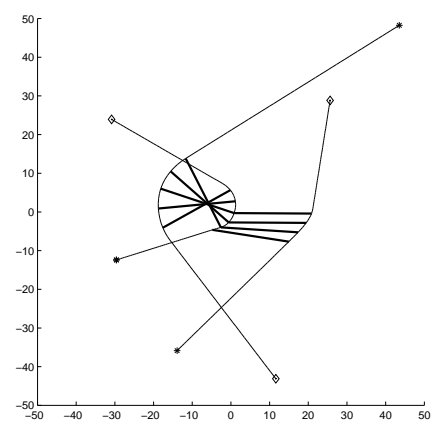

Fig. 6. Concatenation of geodesic segments.

Geodesics in state 8 and 9. Domains $X_{5}, X_{6}$ and $X_{7}$ share a common boundary consisting of two disjoint circles, which form the domains of state 8 and state 9 respectively. In both states, the three agents form an equilateral triangle centered at the origin. The only difference is their orientation. Agents 1, 2 and 3 are numbered counterclockwise in state 8 and clockwise in state 9 .

Consider state 8 and its domain $X_{8} . X_{8}$ is a one dimensional circle and can be parameterized by $\sigma$, which is the angle between the line segment joining the origin to agent 1 and the positive $x$-axis. A geodesic in $X_{8}$ in this coordinate must then be of the form $\sigma(t)=\omega t$ for some constant angular velocity $\omega$.

In summary, we have characterized geodesic segments in each one of the discrete states. By Theorem 1, the minimizing geodesics corresponding to the optimal collision-free maneuvers for the three agents are $C^{1}$ concatenation of such segments. One example of such concatenations is shown in Fig. 6, where the starting and destination positions of the three agents are marked with stars and diamonds respectively. A rod exists between two agents if and only their distance at the corresponding positions is $r$. However, it should be pointed out that the problem of finding when and where the switches between geodesic segments occur remains an open issue. In [11], we propose a numerical procedure to approximate the minimizing geodesics based on the successive optimization of piecewise linear curves in $M$. At each iteration a convex optimization problem is solved. By choosing a small step size for the piecewise linear curves, we can obtain a reasonably good approximation.

\section{Conclusions}

The problem of optimal collision-free maneuvers for multiple agents is formulated and shown to be equivalent to the problem of finding minimizing geodesics in a certain manifold with boundary, which can in turn be interpreted as an optimal control problem for a hybrid system. The solution is given for the two agents case. For the three agents system we derive the dynamics of the segment of optimal maneuver associated to each discrete state. The overall optimal maneuver is shown to be a $C^{1}$ concatenation of such segments. 


\section{References}

1. F. Albrecht and I.D. Berg. Geodesics in Euclidean space with analytic obstacle. Proceedings of the American Mathematics Society, 113(1):201-207, 1991.

2. R. Alexander and S. Alexander. Geodesics in Riemannian manifolds-with-boundary. Indiana University Mathematics Journal, 30(4):481-488, 1981.

3. S.B. Alexander, I.D. Berg, and R.L. Bishop. Cauchy uniqueness in the Riemannian obstacle problem. In Lecture notes in Math., vol.1209, pag.1-7. Springer-Verlag, 1985.

4. A. Bicchi and L. Pallottino. Optimal planning for coordinated vehicles with bounded curvature. In Proc. Workshop on Algorithmic Foundations of Robotics, Dartmouth, 2000 .

5. M.P. de Carmo. Riemannian geometry. Birkhäuser Boston, 1992.

6. N. Durand, J.M. Alliot, and J. Noailles. Automatic aircraft conflict resolution using genetic algorithms. In 11th Annual ACM Conf. on Applied Computing, 1996.

7. M. Erdmann and T. Lozano-Perez. On multiple moving objects (motion planning). Algorithmica, 2(4):477-521, 1987.

8. E. Frazzoli, Z.H. Mao, J.H. Oh, and E. Feron. Resolution of conflicts involving many aircraft via semidefinite programming. AIAA J. of Guidance, Control and Dynamics. To appear.

9. K. Fujimura. Motion planning in dynamic environments. Springer-Verlag, 1991.

10. J. Hu, M. Prandini, and S. Sastry. Optimal coordinated maneuvers for multiple agents moving on a plane. Technical report, Univ. of California at Berkeley, 2001.

11. J. Hu, M. Prandini, and S. Sastry. Optimal maneuver for multiple aircraft conflict resolution: a braid point of view. In IEEE 39th Conf. on Decision and Control, Sydney, Australia, 2000.

12. J. Hu and S. Sastry. Geodesics in manifolds with boundary: a case study. In preparation, 2001.

13. J. Kuchar and L.C. Yang. Survey of conflict detection and resolution modeling methods. In AIAA Guidance, Navig., and Control Conf., New Orleans, LA, 1997.

14. J.-C. Latombe. Robot motion planning. Kluwer Academic Publishers, 1991.

15. S.M. LaValle and S.A. Hutchinson. Optimal motion planning for multiple robots having independent goals. IEEE Trans. on Robotics and Aut., 14(6):912-925, 1998.

16. J. Lygeros, G.J. Pappas, and S. Sastry. An introduction to hybrid systems modeling, analysis and control. In Preprints of the First Nonlinear Control Network Pedagogical School, pag. 307-329, Athens, Greece, 1999.

17. P.K. Menon, G.D. Sweriduk, and B. Sridhar. Optimal strategies for free-flight air traffic conflict resolution. AIAA J. of Guidance, Control and Dynamics, 22(2):202211, 1999.

18. A. Miele, T. Wang, C.S. Chao, and J.B. Dabney. Optimal control of a ship for collision avoidance maneuvers. J. of Optim. Theory and App., 103(3):495-519, 1999.

19. J.W. Milnor. Morse theory. Annals of mathematics studies, 51. Princeton University Press, 1963. Based on lecture notes by M. Spivak and R. Wells.

20. Radio Technical Commission for Aeronautics. Minimum aviation system performance standards for automatic dependent surveillance-broadcast (ADS-B). Technical report, RTCA-186, 1997. DRAFT 4.0.

21. S. Simic, K.H. Johansson, S. Sastry, and J. Lygeros. Towards a geometric theory of hybrid systems. Hybrid Systems: Computations and Control. Third International Workshop, 2000. In Lecture Notes in Computer Science, number 1790.

22. B. Vronov, M. de Berg, A.F. van der Stappen, P. Svestka, and J. Vleugels. Motion planning for multiple robots. Discrete \& Comput. Geometry, 22(4):502-525, 1999. 